\title{
Sentiment Analysis on Hate Speech using Twitter
}

\author{
Athare Sharayu Shivaji \\ Student, M.E. Comp. Engg. \\ V.A.C.O.E. Ahmednagar
}

\author{
Reweskar V. D. \\ Assistant Professor, \\ Department of Comp. Engg \\ V.A.C.O.E Ahmednagar
}

\begin{abstract}
Sentiment analysis is an application of natural language processing. It is also known as emotion extraction or opinion mining. There is a vast growth in use of social networking sites and microblogging websites, by which communication between people from different countries, cultures and psychological and physical backgrounds became more direct, resulting in more and more conflicts about thoughts and speech used between these people. Hate speech can be explained and it can be put into context as the use of aggressive, violent or offensive language, which targets a specific group of people who shares a common property. In India, a lot of hate speech generated posts are placed on social networking sites. So, to block and catch the hate speech generated posts and to avoid necessary conflicts, we thought of introducing a technique using Twitter, as our data source. We will create and analyze datasets that can be used for machine learning. We are going to analyze the negative and positive hate speech tweets which are generated, and use machine learning algorithms to analyze the tweets, to find the correct meaning behind it whether it is offensive or not. In our project we are going to use various techniques such as Stop Words, Lexicon Analysis, Datasets and Machine Learning to Analyze tweets and find out the sentiments behind it. We are also going to use Apache Spark based parallel processing technique to access only the latest tweets and not the old ones which are already being analyzed.
\end{abstract}

\section{Keywords}

Twitter, Sentiment Analysis, Stop words, Lexicon Analysis, Machine Learning, Apache Spark

\section{INTRODUCTION}

Sentiment analysis using tweets is an interesting topic. But as tweets are daily generated in large numbers from all around the world it is not easy to track them. Many of them are fake tweets which are of no use to the user and is wastage of time. To solve this problem many of them have suggested sentiment analysis for a particular topic. To improve the tweet analysis we thought of using big data to analyze tweets in large numbers. But accessing a large number of tweets was not easy. So we thought of using Apache Spark which handles the clusters from its servers and thus save our extra cost of hardware. This big data using Apache spark will help us access the data better and fast. After accessing huge tweets machine learning algorithm SVM will be implemented to get the sentiment of a particular tweet using training and testing datasets.

\section{MOTIVATION}

Hate Speech is considered a world-wide problem that many countries and organizations have been standing up against. Hate speech does not only create tension between people, its impact can also influence buisnesses, or start serious real-life conflicts. Taking in account of such reasons, websites such as Facebook, Youtube and Twitter prohibit the use of hate speech. However, it is always difficult to control and filter all the contents. That is why, hate speech has been subject to some studies, trying to automatically detect it.

Apache spark is currently used by many big companies to access data fast. It makes use of big data to access the data from various clusters of Twitter Server and saves time. So we thought of using this technology in our project to get data from twitter server to access tweets. So what to do with this data we thought of developing a sentiment analysis application to get sentiment behind a tweet whether it is a positive or negative tweet. The main contribution of this paper is here we classify tweets into two different classes where we make distinction between tweets showing hate, and those being just offensive. The two datasets used are positive and negative dataset.

\section{RELATED WORK}

\subsection{Problem Statement}

Today Social Networking Sites (SOCIAL NETWORKING SITES) have become an important part of our day to day life. We share a lot of personal data on these sites. They help us to make the world smaller and integrate like a small village with each other. There are many SOCIAL NETWORKING SITES available today and many more are increasing each day.

Thus a user uses many Social Networking Sites each day and communicate and share data with friends and family. This communication medium gave rise to complex structure whether a user really like the Social Networking Sites which he uses more or he needs another Social Networking Sites other than he uses more.

Thus one of the most famous SOCIAL NETWORKING SITES is TWITTER which is used to share data and post our thoughts and latest buzz upon the internet. The users using TWITTER have increased constantly in the recent years. So the analysis of this SOCIAL NETWORKING SITES may help in answering and predicting many answers.

This online social network is used by billions of people around the world to remain socially connected to their friends, family members, and coworkers through their computers and mobile phones. Twitter asks one question, "What's going on?" Answers must be fewer than 140 characters. A status update message, called a tweet, is often used as a message to friends, family and colleagues. A user can follow other users; that user's followers can read her tweets on a daily basis. A user who is being followed by another user need not reciprocate by following them back, which leaves the links of the network as directed. Since its launch on July 2006, Twitter users have increased dramatically.

Thus this kind of SOCIAL NETWORKING SITES can be used to predict and analyze the large amount of tweets generated and understand the sentiments behind each tweet whether it is positive, negative or neutral. So we thought of 
designing a project to develop a system which helps in analyzing and helping in developing an application for the purpose of sentiment analysis.

\subsection{Goals and Objectives}

The main goals and objectives of the system are to make effective use of Tweeter API and Apache Spark. To remove Stop Words and apply Lexicon Grammar. Generate Training and Testing datasets and apply Machine Learning. Finally to Perform Successful Classification and analyze the results properly.

\subsection{Software Context}

In this section different technology used for implementing this project are explained i.e. netbeans IDE, JDK, and Weka.

\subsubsection{Netbeans}

NetBeans is a software development platform written in Java. The Netbeans Platform allows applications to be developed from software components called modules. Applications based on the NetBeans Platform, including the NetBeans integrated development environment (IDE), can be extended by third party developers. The NetBeans IDE is primarily intended for development in Java, but also supports other languages, in particular PHP, C/C++ and HTML5. NetBeans is cross-platform and runs on Microsoft Windows, Mac OSX, Linux, Solaris and other platforms supporting a compatible JVM.

\subsubsection{Java Development ToolKit}

The Java Development Kit (JDK) is an implementation of either one of the Java SE, JavaEE or JavaME platforms released by Oracle Corporation in the form of a binary product aimed at Java developers on Solaris, Linux, Mac OS $\mathrm{X}$ or Windows. Since the introduction of Java platform, it has been by far the most widely used SDK. On 17 November 2006, Sun announced that it would be released under the GNU General Public License (GPL), thus making it free software. This happened in large part on 8 May 2007, when Sun contributed the source code to the Open JDK.

\subsubsection{Weka}

Weka contains a collection of visualization tools and algorithms for data analysis and predictive modeling, together with graphical user interfaces for easy access to these functions. The original non-Java version of Weka was a Tcl/Tk front-end to (mostly third-party) modeling algorithms implemented in other programming languages, plus data preprocessing utilities in $\mathrm{C}$, and a Makefile-based system for running machine learning experiments. This original version was primarily designed as a tool for analyzing data from agricultural domains, but the more recent fully Java-based version (Weka 3), for which development started in 1997, is now used in many different application areas, in particular for educational purposes and research.

\section{ARCHITECTURAL DESIGN}

The Sentiment Analysis model's main aim is to provide solution for analyzing lakhs of tweets that are generated every second on twitter. It is low cost and efficient system . It includes Apache Spark, Twitter, Preprocessing, Machine learning etc.

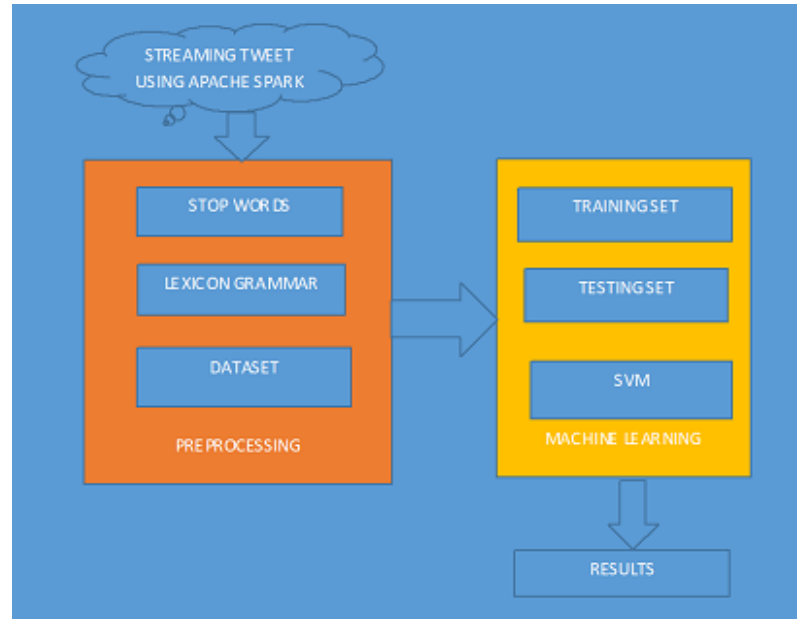

Fig. 1. System Architecture

In proposed system first the tweets are accessed using Apache spark and Twitter4j API, then they are preprocessed and machine learning is applied to it to get the sentiment.

\subsection{Tweet access using Apache Spark Module}

This module first initializes Apache spark over a local ip address and then authentication is provided to twitter by creating a developer account and get authentication details for access. The apache spark will access clusters of twitter and access only the latest tweets.

\subsection{Preprocessing Module}

In this module the tweets will be accessed from text file and then the stop words removal is generated. The stop words which are not good for text mining are matched with an array of stop words and unnecessary words are removed form the tweets. the preprocessed tweets are then stored in a separate text file.

\subsection{Lexicon Grammar Module}

In this module the preprocessed text file is accessed and AFFIN library is applied to it. It returns adjective, noun and pronoun of a word. We only access pronouns and shorten the tweet further. The shorten tweets are again stored in a separate text file.

\subsection{Machine Learning Module}

In this module first we design a training dataset with two classes positive and negative. Then a tweet is accessed and a test dataset is generated. then a instance of SVM classifier is generated and training and testing dataset is applied to it. it returns the result in the form of parameters such as tp rate, $\mathrm{fp}$ rate, precision and recall. we take in to account precision if it is greater than 0.5 then the tweet comes under positive sentiment and if it is smaller than 0.5 the tweet comes under negative sentiment.

\section{MATHEMATICAL MODEL}

This system is "SENTIMENT ANALYSIS OF TWEET USING TWITTER". This system supports only NP-HARD and not NP-COMPLETE. If in this system there is no internet it is case of failure. After following the few algorithmic steps we will achieve the process of a successful Sentiment Analysis. 


\subsection{Set theory applied}

\subsubsection{Preprocessing Module}

Set $(\mathrm{P})=\{\mathrm{P} 0, \mathrm{P} 1, \mathrm{P} 2, \mathrm{P} 3, \mathrm{P} 4, \mathrm{P} 5, \mathrm{P} 6\}$

$\mathrm{P} 0 \in \mathrm{P}=$ Configure Apache Spark

$\mathrm{P} 1 \in \mathrm{P}=$ Authenticate Twitter using Twitter4j library

$\mathrm{P} 2 € \mathrm{P}=$ Enter Keyword

$\mathrm{P} 3 \in \mathrm{P}=$ Dowload Tweets

$\mathrm{P} 4 \mathrm{EP}=$ Remove stop words using BOG(bag of words)

P5EP = Apply Lexicon Grammar

$\mathrm{P} 6 \in \mathrm{P}=$ View Results

\subsubsection{Machine Learning Module}

Set $(\mathrm{M})=\{\mathrm{M} 0, \mathrm{M} 1, \mathrm{M} 2, \mathrm{M} 3, \mathrm{M} 4, \mathrm{M} 5, \mathrm{M} 6, \mathrm{P} 6\}$

$\mathrm{M} 0 \in_{\mathrm{M}}=$ Access preprocessed tweets

$\mathrm{M} 1 \in_{\mathrm{M}}=$ Create a training set

$\mathrm{M} 2 \in \mathrm{M}=$ Generate a testing set

$\mathrm{M} 3 \mathrm{EM}=$ Create $\mathrm{a}$ instance of SVM using Weka

$\mathrm{M} 4 \in_{\mathrm{M}}=$ Train and test SVM

$\mathrm{M} 5 \mathrm{EM}_{\mathrm{M}}=$ get statistics

$\mathrm{M} 6 \mathrm{E}_{\mathrm{M}}=$ fetch precision

$\mathrm{P} 6 \in \mathrm{P}=$ View Results

\subsubsection{Intersection of system}

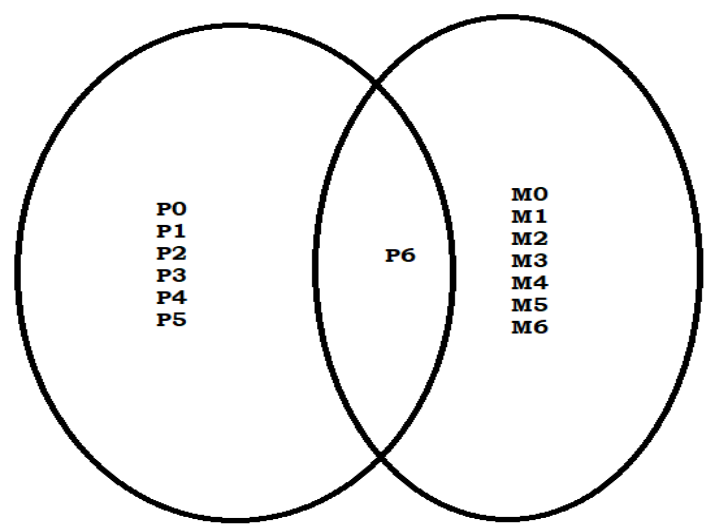

Fig.2 Intersection of probabilities and machine learning

\section{Preprocessing}

We have two possibilities for fetching tweets i.e. whether internet is connected or not.

$\mathrm{P}($ present $)=1 / 2$

$P($ not $)=1 / 2$

Hence, $\mathrm{P}($ tweets $)=\mathrm{P}($ present $)+\mathrm{P}($ not $)$

$=1 / 2+1 / 2$

$=1$

\section{Machine Learning}

We have two possibilities for getting successful sentiment analysis i.e. whether datasets generated are proper or not.

$\mathrm{P}($ present $)=1 / 2$

$\mathrm{P}($ not $)=1 / 2$

Hence, $\mathrm{P}($ dataset $)=\mathrm{P}($ present $)+\mathrm{P}($ not $)$

$=1 / 2+1 / 2$

$=1$

\section{RESULTS AND DISCUSSIONS}

The extraction of features and optimization of parameters is done. The classification is done using the toolkit weka. Weka presents a variety of of classifiers organized into groups based on the type of the algorithm(e.g., decision tree-based, rulebased,etc)[3].Weka contains a collection of visualization tools and algorithms for data analysis and predictive modeling, together with graphical user interfaces for easy access to the functions. To evaluate the performance of classification, different key performance indicators(KPIs) are used which are the percentage of true positives, the precision, the recall.

The following graph shows the results of positive and negative tweets in four attempts.

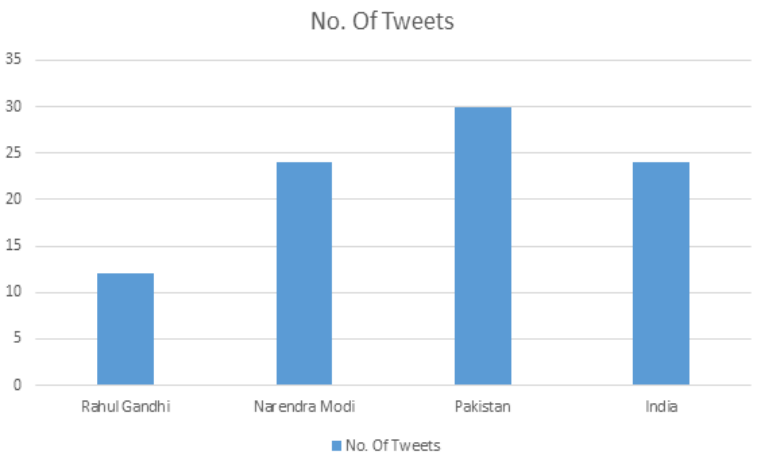

Fig. 3. Tweets retrieved

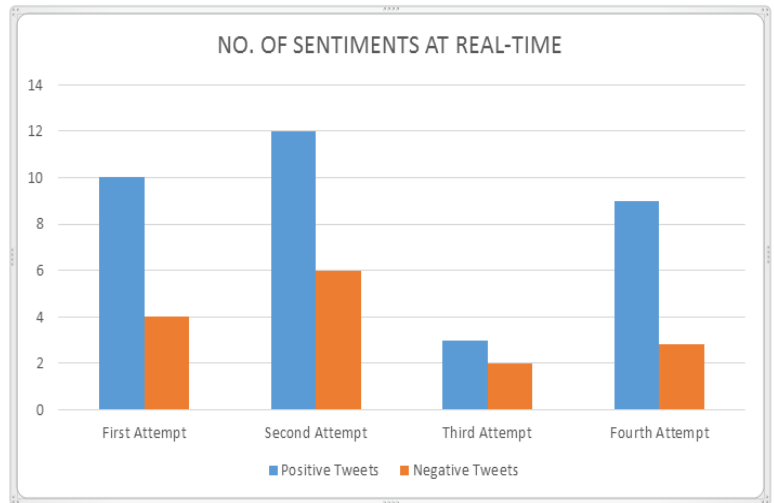

Fig.4 Graph for positive and negative tweets 
NO. OF SENTIMENTS AT REAL-TIME

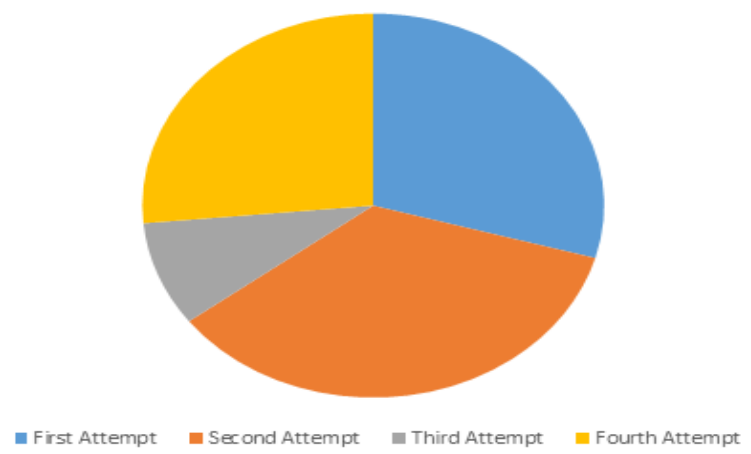

Fig. 5 Pie graph

This system uses Real-time tweets and not offline tweets. It can work on both Offline and Online tweets. It designs a training dataset for each interested word. The training dataset gives perfect prediction than common dataset. Its prediction quality is better than existing system.

\section{CONCLUSION AND FUTURE SCOPE}

In this project, novel sentiment analysis approach using TWITTER and APACHE SPARK together is developed. The basic idea of the project is to use distributed computing in training and testing the machine learning classification. We have assembled various predictions by machine learning algorithms together and viewed the results in three classes such as positive, negative and neutral according to the predictions returned by the system. Our system using Apache spark can be applied to other social networking sites such as Facebook etc.

\section{ACKNOWLEDGMENTS}

We would like to give our sincere thanks to all the staff of V.A.C.O.E Ahmednagar who helped us directly \& indirectly.

\section{REFERENCES}

[1] Yuting $\mathrm{Hu}$, Liang Zheng, Yi Yang, and Yongfen Huang,Twitter100k: A Real-world Dataset for Weakly Supervised Cross-Media Retrieval, IEEE, 2017.

[2] Ana Valdivia, M. Victoria Luzón, and Francisco Herrera,
Sentiment Analysis in TripAdvisor, IEEE, 2017.

[3] Hajime Watanabe, Mondher Bouazizi and Tomoaki Ohtsuki, Hate Speech on Twitter, IEEE, 2018.

[4] Wendy Hall, Ramine Tinati, and Will Jennings, From Brexit to Trump: Social Media's Role in Democracy, IEEE, 2018.

[5] B. Pang and L. Lee, Opinion mining and sentiment analysis, Foundations Trends Inf. Retriev., vol. 2, no. 12, pp. 1135,2008

[6] B. Liu, Sentiment analysis and opinion mining, Synth. Lectures HumanLang. Technol., vol. 5, no. 1, pp. 1167, 2012.

[7] C. Havasi, E. Cambria, B. Schuller, B. Liu, and H. Wang, Knowledgebased approaches to concept-level sentiment analysis, IEEE Intell. Syst., vol. 28, no. 2, pp. 001214, Mar.-Apr. 2013.

[8] C. D. Manning and H. Schtze, Foundations of Statistical Natural Language Processing. Cambridge, MA, USA: MIT Press, 1999.

[9] P. D. Turney, Thumbs up or thumbs down?: Semantic orientation applied to unsupervised classification of reviews, in Proc. ACL, 2002, pp. 417424.

[10] M. Taboada, J. Brooke, M. Tofiloski, K. Voll, and M. Stede, Lexiconbased methods for sentiment analysis, Comput. linguist., vol. 37, no. 2, pp. 267307, 2011.

[11] B. Pang, L. Lee, and S. Vaithyanathan, Thumbs up?: Sentiment classification using machine learning techniques, in Proc. EMNLP, 2002, pp. 7986.

[12] J. Zhao, L. Dong, J. Wu, and K. Xu, Moodlens: An tweets, in SIGKDD, 2012 emoticon-based sentiment analysis system for chinese is used .

[13] A. L. Maas, R. E. Daly, P. T. Pham, D. Huang, A. Y. Ng, and C. Potts, Learning word vectors for sentiment analysis, in Proc. ACL, 2011.

[14] G. Paltoglou and M. Thelwall, A study of information retrieval weighting schemes for sentiment analysis, in Proc. ACL, 2010, pp. 13861395. 